\title{
Atualizações científicas sobre a deficiência de vitamina $D$, de ácido fólico e magnésio
}

\section{na depressão}

\author{
Scientific updates on vitamin D, folic acid and magnesium deficiency in depression \\ Actualizaciones científicas sobre la deficiencia de vitamina D, ácido fólico y magnesio en la \\ depresión
}

Recebido: 01/02/2021 | Revisado: 06/02/2021 | Aceito: 17/02/2021 | Publicado: 25/02/2021

Fabbio Ronnyel Rodrigues Baldoino

ORCID: https://orcid.org/0000-0003-4393-6039 Faculdade de Ciências Humanas, Exatas e da Saúde do Piauí, Brasil Instituto de Educação Superior do Vale do Parnaíba, Brasil E-mail: fabbiobald@gmail.com

Giovanna de Aguiar Mota

ORCID: https://orcid.org/0000-0002-2972-4059 Faculdade de Ciências Humanas, Exatas e da Saúde do Piaú, Brasil Instituto de Educação Superior do Vale do Parnaíba, Brasil E-mail: giovanna_aguiarm@hotmail.com

Myrella Cunha

ORCID: https://orcid.org/0000-0003-1103-9798 Faculdade de Ciências Humanas, Exatas e da Saúde do Piauí, Brasil Instituto de Educação Superior do Vale do Parnaíba, Brasil E-mail: myrellacunhamed@gmail.com

Hellen Pereira Melo

ORCID: https://orcid.org/0000-0003-1813-7577 Faculdade de Ciências Humanas, Exatas e da Saúde do Piauí, Brasil Instituto de Educação Superior do Vale do Parnaíba, Brasil E-mail: hellenmelo1996@gmail.com

Tereza Cristina de Carvalho Souza Garcês

ORCID: https://orcid.org/0000-0001-6337-5166 Faculdade de Ciências Humanas, Exatas e da Saúde do Piauí, Brasil Instituto de Educação Superior do Vale do Parnaíba, Brasil E-mail: tereza.garces@iesvap.edu.br

Ana Rachel Oliveira de Andrade

ORCID: https://orcid.org/0000-0002-8981-0856

Faculdade de Ciências Humanas, Exatas e da Saúde do Piauí, Brasil Instituto de Educação Superior do Vale do Parnaíba, Brasil E-mail: ana.andrade@iesvap.edu.br

\begin{abstract}
Resumo
Objetivo: realizar uma revisão de literatura acerca da influência da deficiência de vitamina $\mathrm{D}$, de metilfolato e de magnésio no desenvolvimento da depressão. Metodologia: Desenvolveu-se uma revisão do tipo integrativa, com estudos publicados entre 2016 e 2020. Inicialmente, estabeleceu-se a questão norteadora, seguida da seleção dos descritores "Deficiência de Vitamina D"; "ácido fólico", "deficiência de magnésio" e "depressão", conforme a plataforma dos Descritores em Ciências da Saúde. As bases de dados destinadas para a busca das publicações, foram: Scielo, Medline, Lilacs e Pubmed. Em seguida definiu-se os critérios exclusão e inclusão das publicações. Resultados e Discussão: Assim, a partir do protocolo metodológico empregado, 24 artigos foram selecionados para compor o estudo. A maior parte $(\mathrm{n}=19,79,17 \%)$ correspondem a publicações que avaliaram de alguma forma a relação da vitamina D com a depressão. Quatro (16,66\%) estudos correspondem à relação do ácido fólico, e um $(4,17 \%)$ ao papel do magnésio nos distúrbios depressivos. Muitos autores observaram que a deficiência de vitamina $\mathrm{D}$ encontra-se associada à presença de sintomas depressivos. Contudo, verificou-se uma escassez de estudos científicos que visem avaliar a influência da deficiência o ácido fólico e magnésio no desenvolvimento da depressão. Conclusão: constatouse que a maior parte estudos concentra-se na associação entre a ausência da vitamina $\mathrm{D}$ e depressão, demonstrando que muitos pesquisadores encaram esse micronutriente como um elemento envolvido em tal distúrbio. Entretanto, ressalta-se a necessidade de mais investigações que visem colaborar com o entendimento do papel do folato e do magnésio nas alterações de humor.
\end{abstract}

Palavras-chave: Deficiência de vitamina D; Ácido fólico; Deficiência de magnésio; Depressão. 


\begin{abstract}
Objective: to carry out a literature review about the influence of vitamin D, methylfolate and magnesium deficiency in the development of depression. Methodology: An integrative review was carried out, with studies published between 2016 and 2020. Initially, the guiding question was established, followed by the selection of the descriptors "Vitamin D deficiency"; "Folic acid", "magnesium deficiency" and "depression", according to the Health Sciences Descriptors platform. The databases for the search for publications were Scielo, Medline, Lilacs and Pubmed. Then, the exclusion and inclusion criteria for publications were defined. Results and Discussion: Thus, based on the methodological protocol employed, 24 articles were selected to compose the study. Most $(n=19,79.17 \%)$ correspond to publications that have somehow evaluated the relationship between vitamin D and depression. Four (16.66\%) studies correspond to the ratio of folic acid, and one $(4.17 \%)$ to the role of magnesium in depressive disorders. Many authors have observed that vitamin D deficiency is associated with the presence of depressive symptoms. However, there was a scarcity of scientific studies aimed at assessing the influence of folic acid and magnesium deficiency on the development of depression. Conclusion: it was found that most studies focus on the association between the absence of vitamin D and depression, showing that many researchers see this micronutrient as an element involved in such a disorder. However, it is emphasized the need for further investigations that aim to collaborate with the understanding of the role of folate and magnesium in mood changes.
\end{abstract}

Keywords: Vitamin D deficiency; Folic acid; Magnesium deficiency; Depression.

\title{
Resumen
}

Objetivo: realizar una revisión de la literatura sobre la influencia de la deficiencia de vitamina D, metilfolato y magnesio en el desarrollo de la depresión. Metodología: Se realizó una revisión integradora, con estudios publicados entre 2016 y 2020. Inicialmente se estableció la pregunta orientadora, seguida de la selección de los descriptores "Deficiencia de vitamina D"; “Ácido fólico", "deficiencia de magnesio" y "depresión”, según la plataforma Health Sciences Descriptors. Las bases de datos para la búsqueda de publicaciones fueron: Scielo, Medline, Lilacs y Pubmed. Luego, se definieron los criterios de exclusión e inclusión de las publicaciones. Resultados y Discusión: Así, en base al protocolo metodológico empleado, se seleccionaron 24 artículos para componer el estudio. La mayoría $(\mathrm{n}=19$, $79,17 \%$ ) corresponden a publicaciones que de alguna manera han evaluado la relación entre vitamina D y depresión. Cuatro $(16,66 \%)$ estudios corresponden a la proporción de ácido fólico y uno $(4,17 \%)$ al papel del magnesio en los trastornos depresivos. Numerosos autores han observado que el déficit de vitamina $\mathrm{D}$ está asociado a la presencia de síntomas depresivos. Sin embargo, hubo una escasez de estudios científicos destinados a evaluar la influencia de la deficiencia de ácido fólico y magnesio en el desarrollo de la depresión. Conclusión: se encontró que la mayoría de los estudios se enfocan en la asociación entre la ausencia de vitamina D y la depresión, mostrando que muchos investigadores ven este micronutriente como un elemento involucrado en tal trastorno. Sin embargo, se enfatiza la necesidad de más investigaciones que tengan como objetivo colaborar con la comprensión del papel del ácido fólico y el magnesio en los cambios de humor.

Palabras clave: Deficiencia de vitamina D; Acido fólico; Deficiencia de magnesio; Depresión.

\section{Introdução}

A depressão representa um importante problema de saúde pública, uma vez que proporciona fortes impactos na saúde geral e psicossocial dos indivíduos, além da sua elevada prevalência entre a sociedade (Gonçalves, et al., 2017). Segundo a Organização Mundial de Saúde, a depressão pode ser definida por quadros de tristeza, perda do interesse e/ou prazer em desempenhar atividades, baixa autoestima e sentimento de culpa, além da constante sensação de cansaço e perda de concentração. A ocorrência de casos mais graves, pode levar o indivíduo ao suicídio (WHO, 2017).

Categoricamente, a depressão pode se apresentar de três formas: depressão menor, que caracteriza-se pelo aparecimento de dois ou mais sintomas, por um período de duas semanas ou mais; distimia, que representa o quadro onde os indivíduos exibem três ou quatro sintomas, por no mínimo, dois anos; e ainda a depressão maior, onde observa-se a presença de um episódio depressivo envolvendo sintomas clássicos como humor deprimido, energia diminuída, perda do interesse e anedonia (Gonçalves, et al., 2017; WHO, 2017).

O problema acomete todas as faixas etárias, e não escolhe raça ou gênero. Embora as investigações apontem para uma maior prevalência entre as mulheres, quando comparada aos homens, muitos estudos já demonstraram a ocorrência de episódios depressivos em ambos os gêneros, em suas mais diversas etapas de vida, como entre mulheres adultas (Gonçalves et al., 2017), homens (Lima, Assunção \& Barreto, 2015), adolescentes (Pasini et al., 2020), puérperas (Hartmann; Mendonza- 
Sassi \& Cesar, 2017), e principalmente, entre a população idosa (Gullich; Duro \& Cesar, 2016; Lima et al., 2016; Teixeira et al., 2016).

No contexto de saúde pública, a depressão apresenta-se como a principal causa de incapacitação, decorrente de uma limitação física, pessoal e social, e que por conseguinte, gera o distanciamento laboral (Lima et al., 2016; Coutinho, 2016). Em 2015, as estimativas apontaram este como um problema presente na vida de 4,4\% da população mundial, sendo mais comum entre as mulheres $(5,1 \%)$, do que entre os homens $(3,6 \%)$. No Brasil, esse número atingiu a marca de 5,8\% da população, totalizando assim 11.548.577 casos de depressão (WHO, 2017).

Embora apresente importantes influências sociais e psicológicas, os distúrbios depressivos também estão relacionados com inúmeros aspectos de ordem biológica e genética. Desta maneira, a depressão pode ser considerada como uma doença multifatorial, com diferentes alterações psicopatológicas, que pode apresentar-se de maneiras distintas em seus sintomas, gravidade, duração e prognóstico. Adicionalmente, considera-se ainda o envolvimento de alterações neuroquímicas (Peron et al., 2004; Lima et al., 2016).

Com base nisso, biologicamente, acredita-se no importante envolvimento dos fatores biológicos, genéticos e psicossociais, de maneira que um influencie a participação do outro. Na etiologia da doença, importantes neurotransmissores encontram-se envolvidos no seu desenvolvimento (Peron et al., 2004). Esses neurotransmissores correspondem às monoaminas como serotonina, dopamina e noradrenalina, as quais determinam efeitos casuísticos na depressão, tendo em vista suas importantes funções no sistema nervoso central. Embora não se tenha explicações claras acerca da fisiopatologia desse distúrbio, os tratamentos farmacológicos são destinados a regulação dos níveis destas aminas (Manji; Drevets \& Charney, 2001; Perito; Fortunato, 2012).

Alguns estudos propõem uma relação mais profunda acerca das causas da depressão, envolvendo, adicionalmente, alterações a nível de sistema endócrino, por meio da diminuição de fatores de crescimento, como o fator neurotrófico derivado do cérebro (do inglês brain-derived neurothophic factor - BDNF) (Drzyzga; Marcinowska \& Obuchowicz, 2009; Perito; Fortunato, 2012). Tal fator pertence à família das neurotrofinas, uma importante classe de proteínas que promovem a sobrevivência e diferenciação neuronal, além de participarem da transmissão sináptica (Fortunato et al. 2009).

Os fatores neurotróficos estão envolvidos em muitas funções celulares e, por meio da ativação de receptores, mantém a neuroplasticidade e saúde celular. Nessa sinalização neurotrófica, a vitamina D tem sido apontada como um importante modulador de tais fatores de crescimento e, desta forma, influencia na sobrevivência e função dos neurônios dopaminérgicos, regulados pelo fator de crescimento de células gliais, e colinérgicos, regulados pelo fator de crescimento neuronal (Kesby et al., 2011; Perito; Fortunato, 2012; Eserian, 2013).

Da mesma forma outros elementos, tais como vitaminas A, C, E e complexo B, além de zinco e magnésio, são elencados como importantes nutrientes que contribuem para o restabelecimento da saúde mental, tanto de indivíduos depressivos como também ansiosos (Rocha, Myva \& Almeida, 2020). Dentre estes, o magnésio é apresentado como um agente que participa de diversas vias envolvidas na regulação do humor, uma vez que contribui ativamente no metabolismo dos receptores de serotonina, dopamina e noradrenalina (Sezini \& Gil, 2014).

Diante do exposto, nota-se a participação de diferentes elementos no desencadeamento dos distúrbios depressivos. Assim, o presente estudo teve como objetivo realizar uma revisão de literatura acerca da influência da deficiência de vitamina $\mathrm{D}$, de metilfolato e de magnésio no desenvolvimento da depressão, a fim de compreender a relação existente entre tais nutrientes e o distúrbio mental. A partir da análise das publicações será possível entender de que maneira todos eles podem estar relacionados com a depressão. 


\section{Metodologia}

Nesse estudou realizou-se uma revisão de literatura integrativa, a partir dos estudos publicados entre os anos de 2016 e 2020. As revisões integrativas são consideradas importantes contribuições acadêmicas que elencam as melhores informações acerca de um assunto, resumindo, sistematicamente, de forma ordenada e abrangente, os resultados alcançados em diversos estudos (Ercole, Melo \& Alcoforado, 2014). As investigações bibliográficas e de natureza qualitativa, são desenvolvidas a partir de um conteúdo já elaborado, tendo como base para sua construção, livros e/ou artigos científicos de renomados autores (Gil, 2010).

Para a elaboração deste estudo, considerou-se o desenvolvido de três importantes etapas. A primeira etapa configurou a delimitação do tema e o questionamento base para a presente investigação. A segunda etapa correspondeu a determinação dos descritores, dos bancos de dados, dos critérios de inclusão e exclusão, além da seleção das publicações. Por fim, a terceira e última etapa, caracterizou-se pela avaliação, interpretação e síntese dos resultados obtidos.

Como questão norteadora determinou-se para a investigação, o seguinte questionamento: "Qual o papel da deficiência de vitamina D, metilfolato e magnésio na depressão?”. Em seguida, com base nos Descritores em Ciências da Saúde - DeCS, selecionou-se as palavras-chave para a busca das publicações: "Deficiência de Vitamina D”; "ácido fólico", “deficiência de magnésio" e "depressão". A Biblioteca Virtual em Saúde (BVS) foi selecionada para a pesquisa das publicações científicas, considerando-se para essa busca as suas bases de dados indexadas: Scientific Electronic Librany Online (Scielo), Literatura Internacional em Ciências da Saúde (MEDLINE), Literatura Latino Americana e do Caribe em Ciências da Saúde (LILACS). Além destas, incluiu-se ainda a National Library of Medicine (PUBMED).

Posteriormente, os critérios para inclusão e exclusão das publicações foram definidos. Para serem incluídos na seleção, apreciou-se os estudos publicados no período estabelecido, artigos disponibilizados nos idiomas português e/ou inglês, trabalhos completos publicados em periódicos; estudos experimentais, relato de casos e/ou ensaios clínicos. Publicações de resumos simples e/ou expandidos, artigos que não apresentaram as informações desejadas, além de artigos duplicados e outras revisões da literatura, foram excluídos da seleção.

As publicações selecionadas foram submetidas a uma avaliação, interpretação, bem como uma síntese das informações obtidas. Por conseguinte, estas são então apresentadas de maneira descritiva, permitindo a observação e descrição das mesmas, reunindo as informações sobre o assunto em tela.

\section{Resultados e Discussão}

Para a elaboração deste trabalho, foram considerados os artigos científicos relacionados ao tema, que permitissem uma compreensão da deficiência destes nutrientes no desenvolvimento da depressão, respondendo desta maneira as indagações que nortearam o levantamento. Assim, após a aplicação dos critérios de inclusão e exclusão, 24 artigos foram selecionados para compor o presente estudo.

Considerando-se o objetivo desta revisão e, com a finalidade de promover um melhor entendimento dos estudos, a reunião das publicações foi realizada separadamente para cada elemento. A Tabela 1 elenca tais publicações, apresentando o título dos manuscritos, autoria e ano de publicação, além do objetivo e principais resultados e conclusões obtidos nos estudos. 
Tabela 1: Artigos publicados entre 2016 e 2020, acerca da relação entre a carência vitamina D, ácido fólico e magnésio no desenvolvimento da depressão.

\begin{tabular}{|c|c|c|}
\hline $\begin{array}{c}\text { Título da } \\
\text { Publicação/Autoria/Ano }\end{array}$ & Objetivo & Principais achados/conclusões \\
\hline $\begin{array}{l}\text { Low vitamin D status is } \\
\text { associated with more depressive } \\
\text { symptoms in Dutch older adults } \\
/ \quad \text { Brouwer- } \\
\text { Brolsma et al., 2016. }\end{array}$ & $\begin{array}{l}\text { Analisar a associação entre os } \\
\text { fatores genéticos relacionados a } \\
\text { vitamina D e o status do próprio } \\
\text { nutriente, com os sintomas } \\
\text { depressivos de } 2.839 \text { pacientes } \\
\text { holandeses idosos. }\end{array}$ & $\begin{array}{l}\text { Foi observada uma associação entre baixos níveis } \\
\text { séricos da 25-hidroxivitamina D (25-OH-D) e os } \\
\text { sintomas da depressão, mas não verificou-se tal } \\
\text { relação entre as concentrações do micronutriente e } \\
\text { sua composição genética. }\end{array}$ \\
\hline $\begin{array}{l}\text { Association between serum 25- } \\
\text { hydroxyvitamin D } \\
\text { Concentrations and depressive } \\
\text { Symptoms in na older Korean } \\
\text { population: A cross-sectional } \\
\text { study/Song et al. } 2016 \text {. }\end{array}$ & $\begin{array}{l}\text { Analisar as associações entre as } \\
\text { concentrações séricas de (25-OH- } \\
\text { D) e sintomas depressivos em } \\
\text { uma população coreana mais } \\
\text { velha. }\end{array}$ & $\begin{array}{l}\text { Verificou-se que concentrações mais baixas de } \\
\text { vitamina D estão associadas de forma } \\
\text { independentemente com os sintomas depressivos na } \\
\text { população estudada. }\end{array}$ \\
\hline $\begin{array}{l}\text { Vitamin D levels and } \\
\text { depressive symptoms in } \\
\text { patients with chronic heart } \\
\text { failure / Johansson et al., } \\
(2016) \text {. }\end{array}$ & $\begin{array}{l}\text { Avaliar a associação do nutriente } \\
\text { com sintomas depressivos, e se } \\
\text { essa associação é mediada pela } \\
\text { função física de pacientes com } \\
\text { insuficiência cardíaca. }\end{array}$ & $\begin{array}{l}\text { Nestes pacientes, ao longo de } 18 \text { meses, um nível } \\
<50 \mathrm{nml} / \mathrm{L} \text { de vitamina } \mathrm{D} \text { foi associado com os } \\
\text { sintomas de distúrbios depressivos, correlacionando- } \\
\text { se de forma significativa com a função física dos } \\
\text { indivíduos. }\end{array}$ \\
\hline
\end{tabular}

\begin{abstract}
Vitamin D deficiency, depression course and mortality: Longitudinal results from The Netherlands study on depression in older persons (NESDO) / Van den Berg et al., 2016.

Sexual function and depressive symptoms in young women with low 2 vitamin D status: a pilot study/Krysiak; Gilowska \& Okopién, 2016.
\end{abstract}

The associations among vitamin
$\mathrm{D}$ deficiency, C-reactive
protein, and depressive
symptom / Shin et al., 2016.
symptom / Shin et al., 2016.
Analisar o efeito dos níveis de vitamina D (25-OH-D e 1,25-OH2D) no curso da depressão e na sua remissão, após dois anos, além do desgaste e mortalidade, em uma população mais velha.

A partir de uma estudo piloto, os autores investigaram a relação da disfunção sexual e os sintomas depressivos, em mulheres jovens com baixo nível de vitamina $\mathrm{D}$.

Avaliar as associações entre os níveis séricos de vitamina $\mathrm{D}$ e proteína $\mathrm{C}$ reativa (PCR) e sintomas da depressão em 52.228 pacientes.

\section{Efficacy of High-Dose supplementation With Oral Vitamin D3 on Depressive Symptoms in Dialysis Patients With Vitamin D3 Insufficiency / Wang et al., 2016.}

Analisar se a suplementação de vitamina D3 em alta dose exerce efeitos benéficos nos sintomas depressivos em 726 pacientes com doença renal, em tratamento com diálise.

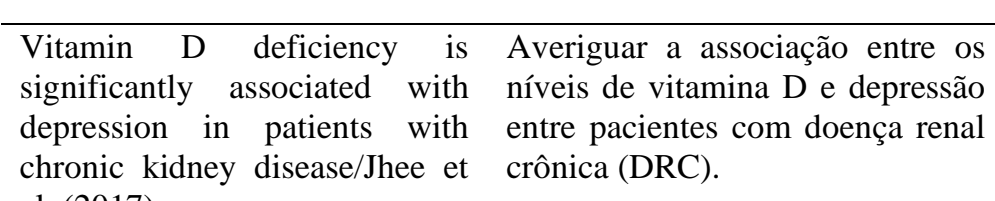
al. (2017).
Não observou-se relação entre os níveis do nutriente e o curso ou a remissão da depressão. Entretanto, indivíduos com deficiência grave de vitamina $\mathrm{D}$, apresentaram uma tendência para uma baixa taxa de remissão. Além disso, níveis extremamente baixos, foram associados à mortalidade.

Observou-se que o baixo nível de vitamina D está associado ao funcionamento sexual feminino anormal, envolvendo falta de desejo, orgasmo e satisfação sexual. Além disso, a gravidade depende desse cenário depende do grau de deficiência do nutriente.

Observou-se uma diferença significativa no nível de vitamina $\mathrm{D}$ entre os indivíduos com e sem sintomas de depressão, contudo não houve diferença no nível de PCR. Ajustes no nível da proteína não alterou a associação entre sintomas depressivos e vitamina D, demonstrando que não há uma relação entre ambos os marcadores na doença.

Em um estudo prospectivo, randomizado e duplo cego, observou-se que a suplementação de vitamina D3 não melhorou de maneira significativa os sintomas. Entretanto, avaliando o efeito da suplementação por tipo de depressão, verificou-se um efeito benéfico em pacientes com depressão vascular em diálise.

Observou-se uma alta prevalência de depressão entre os pacientes com DRC e com deficiência do nutriente, quando comparado com pacientes com DRC sem deficiência de vitamina D. Concluiu-se que o manejo da ausência do micronutriente pode ajudar a prevenir a depressão em tais pacientes. 
Sun Exposure and Behavioral Activation for Hypovitaminosis D and Depression: A Controlled Pilot Study/Thomas \& Al-Anouti, 2017.
Avaliou a eficácia da exposição solar, no aumento dos níveis sérios de 25-OH-D em mulheres com sintomas de depressão.
As mudanças de hábitos, destinadas à realização de atividades ao ar livre e à saída de casa, visando a exposição solar, melhoraram de forma significativa os níveis séricos de 25-OH-D, obtendo efeitos positivos no alívio dos sintomas depressivos.

\begin{abstract}
Vitamin D and symptoms of depression in overweight or obese adults: a cross sectional study and randomized placebocontrolled trial/Mousa et al., 2017.
\end{abstract}

Vitamin D supplementation in bipolar depression: A double blind placebo controlled trial / Marsh, Penny \& Rothschild, 2017.
Examinaram se as concentrações de 25-OH-D estavam associadas a sintomas de depressão e se a suplementação do nutriente reduz tais sintomas, em pessoas obesas e com sobrepeso, com deficiência de vitamina $\mathrm{D}$.

Avaliar o efeito da suplementação de vitamina $D$ em pacientes com depressão bipolar.
A partir da investigação, os autores sugerem que a deficiência de vitamina $\mathrm{D}$ pode não estar relacionada ao risco aumentado de depressão em indivíduos sem depressão clinicamente significativa e que o uso de suplementação do nutriente pode não ser justificado para reduzir os sintomas depressivos na população estudada.

O estudou concluiu que apesar de ter-se obtido um maior aumento nos níveis de vitamina $\mathrm{D}$ no grupo que recebeu a suplementação, não houve redução significativa da diferença nos sintomas depressivos.

\begin{abstract}
Análise de Classes Latentes: um novo olhar sobre o fenômeno depressão em homens idosos no nordeste do Brasil / Leão; Silva \& Moreira, 2017.
\end{abstract}

Identificar a prevalência da Observou-se que baixos níveis séricos de vitamina D depressão em homens idosos e e testosterona estão associados à depressão na fatores associados ao desenvolvimento da doença.

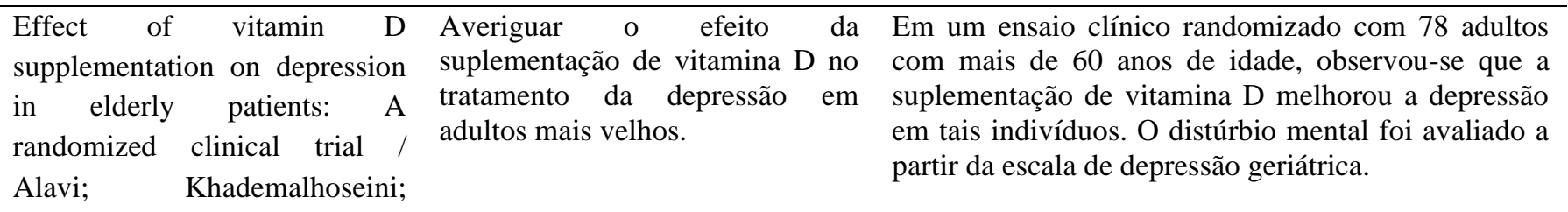

Vakili; Assarian, 2018. população estudada, assim como níveis séricos altos ou normais de TSH também contribuem para uma predisposição à depressão nesses indivíduos.

\footnotetext{
The VITamin D and OmegA-3

TriaL-Depression Endpoint

Prevention (VITAL-DEP):

rationale and design of a large-

scale ancillary study evaluating

vitamin D and marine omega-3

fatty acid supplements for

prevention of late-life

depression / Okereke et al.,

2018.
}

Propor um novo método de estudo envolvendo a suplementação com vitamina D e ômega 3 a longo prazo, e avaliar seus efeitos nos sintomas da depressão e na melhora do humor.

\footnotetext{
Vitamin D Decreases Beck Depression Inventory Score in Patients with Mild to Moderate Ulcerative Colitis: A DoubleBlind Randomized PlaceboControlled Trial / Sharifi et al., 2018.

Dose ranging effects of Vitamin D3 on the Geriatric Depression Score: A Clinical Trial / Yalamanchili \& Gallagher, 2018.
}

O objetivo do estudo foi avaliar o efeito da vitamina D na escala do Beck Depression Inventory - BDI (uma escala para analisar o grau de depressão) em pacientes com colite ulcerativa (CU).

Identificar o efeito do tratamento com várias doses de vitamina $\mathrm{D}$ durante um ano, na escala de depressão geriátrica, em mulheres caucasianas e afro-americanas
O VITAL-DEP representou um método auxiliar de uso em grande escala, e de acompanhamento a longo prazo, através de ensaios clínicos randomizados. O mesmo tem como medida de intervenção e análise, o uso de vitamina D3 (colecacilferol) e óleo de peixe ativo, contendo ômega 3 marinhos. Os autores concluem que o modelo esclarecerá os efeitos de tais suplementos no humor e informará os cuidados clínicos, visando a prevenção da depressão em adultos de meia-idade e idosos. 
mais velhas diagnosticadas com depressão.

\begin{tabular}{lllll}
\hline Effect of Vitamin & D & Determinar o efeito da A análise realizada em dois grupos (suplementação \\
Supplementation on Depressive & suplementação de vitamina D com vitamina D e placebo), demonstrou que os & com \\
Symptoms in Patients With & sobre os sintomas depressivos em & sintomas depressivos melhoraram no grupo que \\
Knee Osteoarthritis/Zheng et pacientes com osteoartrite de & recebeu a vitamina D e que a manutenção dos níveis \\
al., 2018. & joelho e deficiência de vitamina desse nutriente, ao longo de 24 meses, contribui \\
& D. Bem como avaliar se a positivamente para reduzir tais sintomas em pacientes \\
manutenção de vitamina D sérica com osteoartrite.
\end{tabular}
suficiente tem efeitos benéficos sobre tais sintomas.

\begin{tabular}{|c|c|c|}
\hline $\begin{array}{l}\text { Effects of vitamin D } \\
\text { supplementation on depressive } \\
\text { symptoms in type } 2 \text { diabetes } \\
\text { mellitus patients: Randomized }\end{array}$ & $\begin{array}{l}\text { Analisar os efeitos da } \\
\text { monoterapia com vitamina D } \\
\text { (sem drogas antidepressivas) } \\
\text { sobre os sintomas depressivos } \\
\text { (leves e moderados) de pacientes }\end{array}$ & $\begin{array}{l}- \text { se } \\
0 \quad \text { que } \\
0 \quad \text { nas } \\
8 \text { para } \\
\text { anhado } \\
\text { tilizada }\end{array}$ \\
\hline
\end{tabular}
2019.

\begin{tabular}{|c|c|}
\hline $\begin{array}{l}\text { Vitamin D3 supplementation } \\
\text { and treatment outcomes in } \\
\text { patients with depression } \\
\text { (D3-vit-dep) / Hansen et al., } \\
2019 \text {. }\end{array}$ & 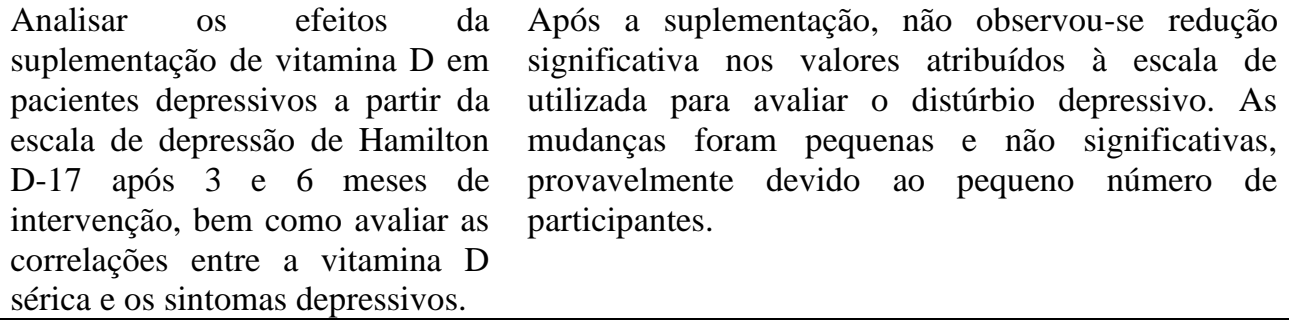 \\
\hline
\end{tabular}

\section{Ácido Fólico}

\begin{abstract}
Augmentation of effect of venlafaxine by folic acid in behavioural paradigms of depression in mice: Evidence of serotonergic andproinflammatory cytokine pathways / Thomas; Khanam \& Vohora, 2016.

Effects of Two-Year Vitamin B12 and Folic Acid Supplementation on Depressive Symptoms and Quality of Life in Older Adults with Elevated Homocysteine Concentrations: Additional Results from the BPROOF Study, an RCT / Koning et al., 2016.

Impact of preconceptional
micronutrient supplementation
on maternal mental health
during pregnancy and
postpartum: results from a
randomized controlled trial in
Vietnam/Nguyen et al., 2017.
\end{abstract}

Effect of Multinutrient Supplementation and FoodRelated Behavioral Activation Therapy on Prevention of Major Depressive Disorder

\begin{abstract}
Avaliar o efeito da combinação da venlafaxina com ácido fólico em modelos de depressão induzida por estresse com foco na via inflamatória.
\end{abstract}

A combinação do ácido fólico com baixas doses da venlafaxina promoveu um aumento no seu efeito antidepressivo, com uma elevação dos níveis de serotonina no cérebro. Assim os autores concluem que tal associação pode ser uma estratégia ideal para aumentar sua eficácia terapêutica, em menores doses.
Avaliar se a suplementação de vitamina B12 e ácido fólico pode reduzir as concentrações de homocisteína, com uma diminuição dos sintomas da depressão, uma vez que o seu aumento encontra-se associado com a presença do distúrbio.
Analisar os efeitos da suplementação pré-concepcional de micronutrientes nos sintomas depressivos maternos, durante a gravidez e o pós-parto.
Em um estudo randomizado, 5.011 mulheres receberam múltiplos micronutrientes, ferro + ácido fólico, ou apenas ácido fólico. Os autores constataram que a suplementação com micronutrientes pré-concepcionais contendo ferro não desempenhou grandes efeitos nos sintomas de depressão, se comparados ao ácido fólico sozinho, que foi mais efetivo.

Os autores observaram que após dois anos, embora a suplementação com os nutrientes tenha sido capaz de reduzir os elevados níveis do aminoácido, o efeito não foi suficiente para reduzir os sintomas depressivos. No entanto, a intervenção proporcionou uma melhora na qualidade de vida geral dos pacientes.

Avaliar o efeito de 2 estratégias nutricionais, incluindo uma suplementação multinutriente dentre eles o ácido fólico, juntamente com terapia de
A suplementação com multinutrientes, comparado ao placebo, e a terapia de ativação comportamental relacionada a alimentos, em comparação com nenhuma terapia, não reduziram os episódios de transtorno depressivo maior. 
Among Overweight or Obese ativação comportamental

Adults With Subsyndromal relacionada a alimentos, bem

Depressive Symptoms

como sua combinação, na

The MooDFOOD Randomized prevenção de um novo episódio

Clinical Trial / Bot et al., 2019. de transtorno depressivo maior,

em adultos com sobrepeso com

sintomas depressivos.

\section{Magnésio}

The Effect of Magnesium

Supplementation on Depression

Status in Depressed Patients

with Magnesium Deficiency: A

Randomized, Double-blind,

Placebo-Controlled Trial/

Rajizadeh et al., 2016.

$\begin{array}{llll}\text { Determinar o efeito } & \text { da } & \text { Os autores observaram que o consumo diário de } \\ \text { suplementação de magnésio no } & \text { comprimidos de óxido de magnésio }(500 \mathrm{mg}) \text { por pelo } \\ \text { estado de depressão de pacientes } & \text { menos } 8 \text { semanas, leva a uma melhora no estado de } \\ \text { deprimidos que sofrem de } & \text { depressão, bem como nos níveis de magnésio. Assim, } \\ \text { deficiência de magnésio. } & \text { concluem que a avaliação do magnésio sérico e a } \\ & \text { resolução dessa deficiência influenciam } \\ & \text { positivamente no tratamento dos pacientes } \\ & \text { depressivos. }\end{array}$

Fonte: Autores (2021).

Como apresentado na Tabela 1, é possível observar que dos 24 estudos selecionados, a maior parte $(\mathrm{n}=19,79,17 \%)$ correspondem a publicações que avaliaram de alguma forma a relação da vitamina D com a depressão. Quatro (16,66\%) estudos correspondem à relação do ácido fólico, e um $(4,17 \%)$ ao papel do magnésio nos distúrbios depressivos. Nota-se, a priori, maiores evidências científicas destinadas a compreender como a vitamina D está relacionada à depressão, especificamente, como a ausência desse nutriente pode contribuir para o estabelecimento do distúrbio mental.

Analisando os tipos de estudos realizados verifica-se que em sua maioria, são ensaios clínicos randomizados. Estes estudos permitem uma melhor análise do problema abordado, pois dentre os vários tipos de estudos experimentais, ele proporciona as mais fortes evidências sobre a análise realizada, em sua maioria, a partir de uma comparação entre um grupo de interesse, que faz uso de uma terapia, e um grupo controle (Oliveira \& Parente, 2010; Oliveira; Velarde \& Sá, 2015). Entretanto, apesar da enorme colaboração destes estudos, nota-se a realização de poucos estudos experimentais, atualmente, envolvendo uma investigação que vise avaliar a via metabólica destes nutrientes na depressão.

Ao analisar as propostas investigativas, observa-se que os estudos consideraram parâmetros estabelecidos por diferentes escalas para avaliar os níveis de depressão entre os participantes. Isso foi uma característica comum entre muitos estudos, o que demonstra que os pesquisadores se preocupam em realizar estudos com designs bem estabelecidos, regidos por critérios e padrões já conhecidos.

No contexto da deficiência de vitamina D os estudos buscaram analisar como a falta desse micronutriente está relacionada aos sintomas da depressão. Muitos autores obtiveram resultados concordantes em observar que tal deficiência encontra-se associada à presença de sintomas depressivos, nos mais diferentes públicos-alvo. Por tais achados é possível inferir que a falta de vitamina $\mathrm{D}$ pode contribuir significativamente para o desenvolvimento do distúrbio depressivo.

Dentre os grupos incluídos nos estudos, encontra-se, principalmente, a população idosa (Brouwer-Brolsma et al., 2016; Song et al. 2016; Alavi; Khademalhoseini; Vakili; Assarian, 2018; Yalamanchili \& Gallagher, 2018), pacientes com insuficiência cardíaca (Johansson et al., 2016); pacientes com doença renal (Wang et al., 2016; Jhee et al., 2017); além de pessoas obesas e com sobrepeso (Mousa et al., 2017); pacientes com colite ulcerativa (Sharifi et al., 2018); pacientes com osteoartrite de joelho (Zheng et al., 2018); pacientes diabéticos (Omidian et al., 2019), dentre outros. As investigações realizadas com tais grupos justifica-se pelo fato de que a depressão representa um problema associado a inúmeras doenças crônicas (Nóbrega; Leal; Marques \& Vieira, 2015). 
A análise dos níveis séricos da 25-hidroxivitamina-D (25-OH-D) foi um parâmetro comum avaliado por muitos pesquisadores, e a partir deste, muitos deles puderam observar que a diminuição em tais níveis está relacionada com a presença de sintomas depressivos (Brouwer-Brolsma et al., 2016; Song et al. 2016; Johansson et al., 2016; Thomas \& Al-Anouti, 2017). A exemplos disso, Jhee et al. (2017) consideraram como deficiência de vitamina D um nível sérico $\leq 10 \mathrm{mg} / \mathrm{mL}$ de 25 hidroxivitamina D3. No estudo, os autores observaram uma relação existente entre uma alta prevalência de depressão em pacientes com doença renal crônica e com deficiência do nutriente, quando comparado com o mesmo grupo de pacientes sem deficiência de vitamina D. Para os pesquisadores o manejo da ausência do micronutriente pode ajudar a prevenir a depressão nestes indivíduos.

Dentre todos os estudos encontrados, um único estudo brasileiro foi localizado. O trabalho realizado por Leão, Silva e Moreira (2017) mostrou que baixos níveis de vitamina D e testosterona estavam associados com sintomas de depressão em uma população de 162 pacientes atendidos na rede atenção básica em Recife, Pernambuco. Os autores também observaram que níveis séricos altos ou normais de TSH (hormônio tireoestimulante) também contribuem para uma predisposição à depressão nesses indivíduos.

Cabe destacar os efeitos promissores das investigações realizadas por alguns estudos, como o de Thomas e Al-Anouti (2017) que apresentam os efeitos da contribuição da exposição solar no aumento dos níveis séricos de 25-OH-D em mulheres depressivas. $\mathrm{O}$ estudo demonstra que mudanças comportamentais destinadas à prática de atividades ao ar livre, bem como a saída de casa, visando a exposição ao sol, permitem uma elevação na concentração de vitamina $\mathrm{D}$, e esta por sua vez reduz os sintomas característicos da depressão. Adicionalmente, o estudo de Omidian et al. (2019) concluiu que a suplementação de vitamina $\mathrm{D}$ em pacientes com diabetes mellitus do tipo 2, pode protegê-los contra o início de um quadro de depressão maior.

Embora alguns estudos tenham apresentado resultados positivos acerca da associação entre deficiência de vitamina D e depressão, em outras investigações essa relação não foi evidenciada (Van den Berg et al., 2016; Wang et al., 2016; Mousa et al., 2017). Essas diferenças obtidas nas investigações podem ser atribuídas às limitações de cada estudo. De forma semelhante, Hansen et al. (2019) constataram que a intervenção não reduziu os valores da escala utilizada para avaliar os sintomas depressivos na população estudada. Os autores argumentam que embora todos os participantes tenham tido diagnóstico de depressão, o tamanho da população era muito pequeno para determinar se a vitamina $\mathrm{D}$ é útil em uma população recebendo tratamento padrão eficaz para a depressão.

Como observado na maioria dos estudos científicos envolvendo a vitamina $\mathrm{D}$, as investigações tiverem como finalidade avaliar efeitos da suplementação com este micronutriente. Entretanto, o estudo de Okereke et al. (2018) destaca-se por apresentar um modelo de estudo baseado no desenvolvimento de um método que permita avaliar efeitos de uma suplementação com vitamina D e ômega 3, em grande escala e a longo prazo. Os autores apresentam que o método proposto inclui características importantes para estudos modernos de prevenção, envolvendo ensaios clínicos, tais como a integração extensiva de moderadores biológicos plausíveis e a avaliação dos efeitos.

Nesse estudo, identificou-se apenas quatro publicações que explanam a relação entre ácido fólico e depressão. E nesse sentido destaca-se que apenas dois deles apresentam resultados satisfatórios acerca desta relação, demonstrado através de uma abordagem combinada com ácido fólico e baixas doses de um antidepressivo padrão. Observou-se que a associação com ácido fólico capaz de melhorar o efeito da venlafaxina, em baixas doses (Thomas; Khanam \& Vohora, 2016). Por outro lado, Vietnam/Nguyen et al. (2017) observaram que a suplementação apenas com ácido fólico, em mulheres grávidas, melhorou os sintomas depressivos, se comparado com uma suplementação rica em ácido fólico e ferro.

Os critérios estabelecidos para a seleção das publicações utilizadas nesta revisão de literatura, permitiu localizar apenas um único artigo científico que trata da deficiência de magnésio e o desenvolvimento da depressão. O estudo de Rajizadeh et al. (2016) constatou que o uso de 500mg óxido de magnésio, diariamente, por um período de oito semanas, 
promove uma melhora no estado de depressão, em pacientes com baixos níveis de magnésio. Para os autores a investigação das concentrações séricas desse elemento, seguida da suplementação, contribui de maneira positiva para o tratamento.

É notório que, nos últimos cinco anos, muitas investigações foram realizadas buscando conhecer e esclarecer os efeitos da ausência de vitamina D no organismo, e como essa característica pode contribuir para a presença dos sintomas da depressão, em diversos grupos de pacientes. Por outro lado, chama-se a atenção para a escassez de estudos científicos que visem avaliar a influência da deficiência dos outros nutrientes aqui estudados, para o desenvolvimento da depressão (Tabela 1). Com isso, percebe-se a necessidade de mais investigações que possam compreender as relações existentes entre os demais micronutrientes e a depressão. A realização de novos ensaios poderá contribuir para o esclarecimento do papel do ácido fólico e do magnésio no desenvolvimento dos sintomas depressivos, colaborando desta maneira para uma real compreensão do metabolismo destes elementos.

\section{Conclusão}

Uma vez que a depressão representa uma das principais causas de morte em todo o mundo, a constante realização de estudos sobre esta temática é de grande relevância. Assim, considerando-se o objetivo desta revisão de literatura, a manutenção de informações atualizadas contribui positivamente para uma compreensão deste problema de saúde pública. Neste sentido, integrou-se as principais publicações científicas destinadas às investigações da relação entre deficiência de vitamina D, ácido fólico, magnésio e depressão, disponibilizadas nos últimos cincos anos.

Foi possível constatar que a maior parte estudos buscaram conhecer a associação entre a carência da vitamina D e o distúrbio depressivo. Isso demonstra que muitos pesquisadores já encaram esse micronutriente como um importante elemento envolvido nas alterações de humor dos indivíduos. Ao passo que se percebe a grande proporção de estudos acerca da primeira associação, ressalta-se a necessidade de mais investigações que visem colaborar com o entendimento do papel do folato e do magnésio nos transtornos depressivos, permitindo o conhecimento da vida metabólica das alterações psicopatológicas.

A realização de novos estudos poderá contribuir ainda mais para a compreensão desse problema, destacando a necessidade de se entender o envolvimento do ácido fólico e magnésio na depressão e desta forma, proporcionar novos conhecimentos na área.

\section{Referências}

Alavi, N. M., Khademalhoseini, S., Vakili, Z., \& Assarian, F. (2019). Effect of vitamin D supplementation on depression in elderly patients: A randomized clinical trial. Clinical Nutrition, 38(5), 2065-2070. https://www.sciencedirect.com/science/article/abs/pii/S026156141832449X. https://doi.org/10.1016/j.clnu.2018.09.011.

Bot, M., Brouwer, I. A., Roca, Miquel., Kohls, E., Penninx, B. W. J. H., Watkins, E. et al. (2019). Effect of multinutrient supplementation and food-related behavioral activation therapy on prevention of major depressive disorder among overweight or obese adults with subsyndromal depressive symptoms: the MooDFOOD randomized clinical trial. Jama, 321(9), 858-868. https://jamanetwork.com/journals/jama/article-abstract/2726983. doi: 10.1001/jama.2019.0556

Brouwer-Brolsma, E. M., Dhonukshe-Rutten, R. A. M., Van Wijngaarden, J. P., Van Der Zwaluw, N. L., Sohl, E., In’t Veld, P. H., Van Dijk, S. C., et al. (2016). Low vitamin D status is associated with more depressive symptoms in Dutch older adults. European journal of nutrition, $55(4), 1525-1534$. https://link.springer.com/article/10.1007/s00394-015-0970-6. 10.1007/s00394-015-0970-6.

Coutinho, M. P. L., Pinto, A. V. L., Cavalcanti, J. G., Araújo, L. S., \& Coutinho, M. L. (2016). Relação entre depressão e qualidade de vida de adolescentes no contexto escolar. Psicologia, Saúde \& Doenças, 17(3), 338-351. http://www.scielo.mec.pt/scielo.php?pid=S1645-

00862016000300003\&script=sci_arttext\&tlng=es. http://dx.doi.org/10.15309/16psd170303.

Drzyzga, L. R., Marcinowska, A., \& Obuchowicz, E. (2009). Antiapoptotic and neurotrophic effects of antidepressants: A review of clinical and experimental studies. Brain Research Bulletin, 79(5), 248-257. https://www.sciencedirect.com/science/article/abs/pii/S0361923009001002.

10.1016/j.brainresbull.2009.03.009.

Ercole, F. F., Melo, L. S., \& Alcoforado, C. L. G. C. (2014). Revisão integrativa versus revisão sistemática. Revista Mineira de Enfermagem, 18(1), 9-12. http://www.reme.org.br/artigo/detalhes/904. 10.5935/1415-2762.20140001.

Eserian, J. K. (2013). Papel da vitamina D no estabelecimento e tratamento de transtornos neuropsiquiátricos. Revista de Ciências Médicas e Biológicas, 12(2), 234-238. https://cienciasmedicasbiologicas.ufba.br/index.php/cmbio/article/view/6989. http://dx.doi.org/10.9771/cmbio.v12i2.6989. 
Fortunato, J. J., Réus, G. Z., Kirsch, T. R., Stringari, R. B., Stertz, L., Kapczinski, F. et al. (2009). Acute harmine administration induces antidepressive-like effects and increases BDNF levels in the rat hippocampus. Progress in Neuro-Psychopharmacology and Biological Psychiatry, 33(8), 1425-1430. https://www.sciencedirect.com/science/article/abs/pii/S0278584609002498. 10.1016/j.pnpbp.2009.07.021.

Gil, A. C. (2010) Como elaborar projetos de pesquisa. (5 $5^{\mathrm{a}}$ ed.): Atlas.

Gonçalves, A. M. C., Teixeira, M. T. B., Gama, J. R. A., Lopes, C. S, Silva, G. A., Gamarra, C. J., et al. Prevalência de depressão e fatores associados em mulheres atendidas pela Estratégia de Saúde da Família. (2018). Jornal Brasileiro de Psiquiatria 67(2), 101-109. https://www.scielo.br/scielo.php?pid=S0047$20852018000200101 \&$ script=sci_arttext\&tlng=pt. doi.org/10.1590/0047-2085000000192.

Gullich, I., Duro, S. M. S., \& Cesar, J. A. (2016). Depressão entre idosos: um estudo de base populacional no Sul do Brasil. Revista Brasileira de Epidemiologia, 19(4), 691-701. https://www.scielosp.org/article/rbepid/2016.v19n4/691-701/. https://doi.org/10.1590/1980-5497201600040001.

Hansen, J. P. Pareek, M., Hvolby, A., Schmedes, A., Toft, Tomas, Dahl, E., \& Nielsen, C. T. (2019). Vitamin D3 supplementation and treatment outcomes in patients with depression (D3-vit-dep). BMC research notes, 12(1), 1-6. https://www.ncbi.nlm.nih.gov/pmc/articles/PMC6446320/. 10.1186/s13104-019-4218-

Hartmann, J. M., Mendoza-Sassi, R. A., \& Cesar, J. A. (2017). Depressão entre puérperas: prevalência e fatores associados. Cadernos de Saúde Pública, 33(9), e00094016. https://www.scielosp.org/article/csp/2017.v33n9/e00094016/. 10.1590/0102-311X00094016

Jhee, J. H., Kim, H., Seohyun, P., Yun, H. R., Su-Young, J., Kee, Y. K., Et Al., (2017). Vitamin D deficiency is significantly associated with depression in patients with chronic kidney disease. PLoS One, 12 (2), e0171009. https://journals.plos.org/plosone/article?id=10.1371/journal.pone.0171009. 10.1371/journal.pone.0171009.

Johansson, P., Alehagen, U., Van Der Wal, M. H. L., Svensson, E., \& Jaarsma, T. (2016). Vitamin D levels and depressive symptoms in patients with chronic heart failure. International journal of cardiology, 207, 185-189. https://www.sciencedirect.com/science/article/abs/pii/S0167527316301723. https://doi.org/10.1016/j.ijcard.2016.01.173.

Kesby, J. P., Eyles, D. W., Burne, T. H.J., \& McGrath, J. J. (2011). The effects of vitamin D on brain development and adult brain function. Molecular and cellular endocrinology, 347(1-2), 121-127. https://www.sciencedirect.com/science/article/abs/pii/S0303720711002565. 10.1016/j.mce.2011.05.014.

Koning, E. J., Van Der Zwaluw, N. L., Van Wijngaarden, J. P., Sohl, E., Brouwer-Brolsma, E. M., Van Marwijk, H. W. J. et al. (2016). Effects of two-year vitamin B12 and folic acid supplementation on depressive symptoms and quality of life in older adults with elevated homocysteine concentrations: additional results from the B-PROOF study, an RCT. Nutrients, 8(11), 1-16. https://www.mdpi.com/2072-6643/8/11/748. doi:10.3390/nu8110748.

Krysiak, R., Gilowska, M., \& Okopień, B. Sexual function and depressive symptoms in young women with low vitamin D status: a pilot study. (2016). European Journal of Obstetrics \& Gynecology and Reproductive Biology, 204, 108-112,

https://www.sciencedirect.com/science/article/abs/pii/S030121151630849. http://dx.doi.org/10.1016/j.ejogrb.2016.08.001

Leão, R. C. H., Silva, V. L., \& Moreira, R. S. (2017). Análise de Classes Latentes: um novo olhar sobre o fenômeno depressão em homens idosos no nordeste do Brasil. Revista Brasileira de Geriatria e Gerontologia, 20(6), 814-825. https://www.scielo.br/scielo.php?pid=S1809-

98232017000600814\&script=sci_arttext\&tlng=pt. https://doi.org/10.1590/1981-22562017020.160159.

Lima, A. M. P., Ramos, J. L. S., Bezerra, I. M. P., Rocha, R. P. B., Batista, H. M. T., \& Pinheiro, W. R. (2016). Depressão em idosos: uma revisão sistemática da literatura. Revista de Epidemiologia e Controle de Infecção, 6(2), 97-103.

https://pdfs.semanticscholar.org/bad9/3826244c85d7fcce2ac412a34c8f3b4dcffd.pdf. http://dx.doi.org/10.17058/reci.v6i2.6427.

Lima, E. P., Assunção, A. A., \& Barreto, S. M. Prevalência de depressão em bombeiros. (2015). Cadernos de Saúde Pública, 31, 733-743 https://www.scielosp.org/article/csp/2015.v31n4/733-743/. 10.1590/0047-2085000000192.

Manji, H. K., Drevets, W. C., \& Charney, D. S. (2001). The cellular neurobiology of depression. Nature medicine, 7(5), 541-547.

https://www.nature.com/articles/nm0501_541.

Marsh, W. K., Penny, J. L., \& Rothschild, A. J. (2017). Vitamin D supplementation in bipolar depression: A double blind placebo controlled trial. Journal of psychiatric research, 95, 48-53. https://www.sciencedirect.com/science/article/abs/pii/S002239561730095X. 10.1016/j.jpsychires.2017.07.021.

Mousa, A., Naderpoor, N., Courten, M. P.J., \& Courten, B. (2018). Vitamin D and symptoms of depression in overweight or obese adults: A cross-sectional study and randomized placebo-controlled trial. The Journal of steroid biochemistry and molecular biology, 177, $200-208$.

https://www.sciencedirect.com/science/article/abs/pii/S0960076017302169. http://dx.doi.org/doi:10.1016/j.jsbmb.2017.08.002.

Nguyen, Phuong H., Digirolamo, A. M., Gonzalez-Casanova, I., Pham, H., Hao, W., Nguyen, H., Truong, T. V., et al. (2017). Impact of preconceptional micronutrient supplementation on maternal mental health during pregnancy and postpartum: results from a randomized controlled trial in Vietnam. $B M C$ women's health, 17(1), 1-9. https://link.springer.com/article/10.1186/s12905-017-0401-3. 10.1186/s12905-017-0401-3.

Okereke, O. I. Reynolds, C. F., Mischoulon, D., Chang, G., Cook, N. R., Copeland, Trisha, Friedenberg, G. et al., (2018). The VITamin D and OmegA-3 TriaL-Depression Endpoint Prevention (VITAL-DEP): rationale and design of a large-scale ancillary study evaluating vitamin D and marine omega-3 fatty acid supplements for prevention of late-life depression. Contemporary clinical trials, 68, 133-145. https://www.sciencedirect.com/science/article/pii/S1551714417306353. 10.1016/j.cct.2018.02.017.

Oliveira, M. A. P., Velarde, L. G. C., \& Sá, R. A. M. (2015). Ensaios clínicos randomizados: Série Entendendo a Pesquisa Clínica 2. Femina, 43(1). http://files.bvs.br/upload/S/0100-7254/2015/v43n1/a4842.pdf.

Omidian, M., Mahmoudi, M., Abshirini, M., Eshraghian, M., Reza, J. M. H., Zarei, M., Hasani, H., \& Djalali, M. (2019). Effects of Vitamin D supplementation on depressive symptoms in type 2 diabetes mellitus patients: Randomized placebo-controlled double-blind clinical trial. Diabetes \& Metabolic Syndrome: Clinical Research \& Reviews, 13, (4), 2375-2380. https://www.sciencedirect.com/science/article/abs/pii/S1871402119303236. https://doi.org/10.1016/j.dsx.2019.06.011. 
Pasini, A. L. W., Silveira, F. L., Silveira, G. B., Busatto, J. H., Pinheiro, J. M., Leal, T. G. et al. Suicídio e depressão na adolescência: fatores de risco e estratégias de prevenção. Research, Society and Development, 9(4), e36942767-e36942767, 2020. https://rsdjournal.org/index.php/rsd/article/view/2767. http://dx.doi.org/10.33448/rsd-v9i4.2767

Perito, M. E. S., Fortunato, J. J. (2012). Marcadores Biológicos da Depressão. Revista Neurociencias, $20(4), 597-603$. https://periodicos.unifesp.br/index.php/neurociencias/article/view/8235/5766.

Peron, A. P., Neves, G. Y. S., Brandão, M., \& Vicentini. V. E. P. (2004). Aspectos biológicos e sociais da depressão. Arquivos de Ciências da Saúde da UNIPAR, 8(1). https://revistas.unipar.br/index.php/saude/article/viewFile/240/213.

Rajizadeh, A., Mozaffari-Khosravi, H., Yassini-Ardakani, M., \& Dehghani, A. (2017). Effect of magnesium supplementation on depression status in depressed patients with magnesium deficiency: A randomized, double-blind, placebo-controlled trial. Nutrition, 35, 56-60. https://www.sciencedirect.com/science/article/abs/pii/S0899900716302441. https://doi.org/10.1016/j.nut.2016.10.014

Rocha, A. C. B., Myva, L. M. M., \& Almeida, S. G. (2020). O papel da alimentação no tratamento do transtorno de ansiedade e depressão. Research, Society and Development, 9(9), e724997890-e724997890. https://rsdjournal.org/index.php/rsd/article/view/7890. https://doi.org/10.33448/rsd-v9i9.7890.

Sezini, A. M., \& Do Coutto Gil, C. S. G. (2014). Nutrientes e depressão. Vita et Sanitas, 8(1), 39-57.

http://fug.edu.br/revistas/index.php/VitaetSanitas/article/view/29.

Sharifi, A., Vahedi, H., Nedjat, S., Mohamadkhani, A., \& Attar, M. J. H. (2019). Vitamin D Decreases Beck Depression Inventory Score in Patients with Mild to Moderate Ulcerative Colitis: A Double-Blind Randomized Placebo-Controlled Trial. Journal of Dietary Supplements, 16(5), 541-549. https://www.tandfonline.com/doi/abs/10.1080/19390211.2018.1472168. https://doi.org/10.1080/19390211.2018.1472168

Shin, Y. C., Jung, C. H., Kim, H. J., Kim, E. J. \&Lim, S. W. (2016). The associations among vitamin D deficiency, C-reactive protein, and depressive symptoms. Journal of Psychosomatic Research, 90, p. 98-104. https://www.sciencedirect.com/science/article/abs/pii/S0022399916304184. https://doi.org/10.1016/j.jpsychores.2016.10.001.

Song, B. M., Kim, H. C., Rhee, Y., Youm, Y., \& Kim, C. O. (2016). Association between serum 25-hydroxyvitamin D concentrations and depressive symptoms in an older Korean population: a cross-sectional study. Journal of affective disorders, 189, 357-364. https://www.sciencedirect.com/science/article/abs/pii/S016503271530481X. https://doi.org/10.1016/j.jad.2015.09.043.

Teixeira, C. M., Nunes, F. M. S., Ribeiro, F. M. S., Arbinaga, F., \& Vasconcelos-Raposo, J. (2016). Atividade física, autoestima e depressão em idosos. Cuadernos de Psicología del deporte, 16(3), 55-66. https://revistas.um.es/cpd/article/view/278431/205461.

Thomas, J., \& Al-Anouti, F. (2018). Sun Exposure and Behavioral Activation for Hypovitaminosis D and Depression: a controlled pilot study. Community Mental Health Journal, 54(6), 860-865. https://link.springer.com/article/10.1007/s10597-017-0209-5. https://doi.org/10.1007/s10597-017-0209-5.

Thomas, J., Khanam, R., \& Vohora, D. (2016). Augmentation of effect of venlafaxine by folic acid in behavioral paradigms of depression in mice: Evidence of serotonergic and pro-inflammatory cytokine pathways. Pharmacological Reports, 68(2), 396-403.

https://link.springer.com/article/10.1016/j.pharep.2015.10.003. http://dx.doi.org/10.1016/j.pharep.2015.10.003.

Van Den Berg, K. S., Marijnissen, R. M., Van Den Brink, R. H. S., Naarding, P., Comijs, H. C., \& Voshaar, R. C. O. (2016). Vitamin D deficiency, depression course and mortality: longitudinal results from the Netherlands Study on Depression in Older persons (NESDO). Journal of Psychosomatic Research, 83, p. 50-56. https://www.sciencedirect.com/science/article/abs/pii/S0022399916300393. https://doi.org/10.1016/j.jpsychores.2016.03.004.

Wang, Y., Liu, Y., Lian, Y., Li, N., Liu, H., \& Li, G. (2016). Efficacy of high-dose supplementation with oral vitamin D3 on depressive symptoms in dialysis patients with vitamin D3 insufficiency: a prospective, randomized, double-blind study. Journal of Clinical Psychopharmacology, 36(3), 229-235. https://journals.lww.com/psychopharmacology/Abstract/2016/06000/Efficacy_of_High_Dose_Supplementation_With_Oral.7.aspx. 10.1097/JCP.0000000000000486.

WHO. (2017). Depression and Other Common Mental Disorders: Global Health Estimates. Geneva: World Health Organization. Licence: CC BY-NC-SA. 3.0 IGO. https://apps.who.int/iris/bitstream/handle/10665/254610/WHO-MSD-MER-2017.2eng.pdf;jsessionid=6BE6FA7DA1B03890BFF2F148B11FB047?sequence $=1$.

Yalamanchili, V., \& Gallagher, J. C. (2018). Dose ranging effects of vitamin D3 on the geriatric depression score: A clinical trial. The Journal of steroid biochemistry and molecular biology, 178, p. 60-64. https://www.sciencedirect.com/science/article/abs/pii/S0960076017303254. https://doi.org/10.1016/j.jsbmb.2017.10.025.

Zheng, S., Tu, L., Cicuttini, F., Han, W., Zhu, Z., Antony, B., Wluka, A., et al. (2019). Effect of vitamin D supplementation on depressive symptoms in patients with knee osteoarthritis. Journal of the American Medical Directors Association, 20(12), 1634-1640. https://www.sciencedirect.com/science/article/abs/pii/S1525861018304973. https://doi.org/10.1016/j.jamda.2018.09.006. 